\title{
Quarks, Hadrons, and Emergent Spacetime
}

\author{
Piotr Żenczykowski ${ }^{1}$
}

Published online: 10 August 2018

(C) The Author(s) 2018

\begin{abstract}
It is argued that important information on the emergence of space is hidden at the quark/ hadron level. The arguments follow from the acceptance of the conception that space is an attribute of matter. They involve in particular the discussion of possibly relevant mass and distance scales, the generalization of the concept of mass as suggested by the phase-spacebased explanation of the rishon model, and the phenomenological conclusions on the structure of excited baryons that are implied by baryon spectroscopy. A counterpart of the Eddington-Weinberg relation concerning Regge towers of hadronic resonances is noted.
\end{abstract}

Keywords Planck and hadronic scales · Quantized and emergent space · Rishons · Quarks and hadrons

\section{Introduction}

Modern fundamental physics is dominated by two divergent lines of philosophical thought concerning the notion of space. Both these lines originated in ancient Greece. They have led us to two disjoint but very successful theories that describe the behaviour of matter 'in the small' and 'in the large', the standard model (SM) and general relativity (GR). The SM, a relativistic quantum field theory of interacting elemantary particles, is a descendant of the Democritean line of thought which accepts separation of matter and space, and views space as a mere container in which indivisible 'atoms' move. General relativity is a remote heir of Aristotle's way of thinking in which space (or rather 'place') is regarded as an attribute of matter. Such a logical priority of matter over space is a cornerstone of the views of many modern philosophically-minded thinkers such as Leibniz or Mach and contributed to Einstein's development of GR.

Now, if one accepts the latter position, i.e. the philosophically very attractive view that 'space and time are (...) stretched out by matter' (Heisenberg 1979), that 'objects make space', that without matter there is no space, properties of space should follow those of matter. Accordingly, quantum properties of matter and the discretization of mass prompt us to expect some form of discretization (or quantization) of space. Such arguments and

Piotr Żenczykowski

piotr.zenczykowski@ifj.edu.pl

1 Division of Theoretical Physics, The Henryk Niewodniczański Institute of Nuclear Physics, Polish Academy of Sciences, Radzikowskiego 152, 31-342 Kraków, Poland 
the general wish to unite the classical and quantum aspects of reality lead to the idea of quantum gravity, according to which the macroscopic classical continuous space is to be replaced in the microcosm by some form of quantized space. A simple dimensional argument which involves the gravitational, quantum and relativistic constants $G=6.67 \times 10^{-8} \mathrm{~cm}^{3} /\left(\mathrm{g} \mathrm{s}^{2}\right), h=6.62 \times 10^{-27} \mathrm{~g} \mathrm{~cm}^{2} / \mathrm{s}$, and $c=3 \times 10^{10} \mathrm{~cm} / \mathrm{s}$, and singles out the Planck units of length $l_{P}=\sqrt{h G / c^{3}}=4.05 \times 10^{-33} \mathrm{~cm}$ and time $t_{P}=l_{P} / c$ (as well as mass $m_{P}=\sqrt{h c / G}=5.46 \times 10^{-5} \mathrm{~g}$ ), is then widely accepted as providing the distance and time scales at which the quantum nature of space should manifest.

One of the problems associated with the idea of the emergence of macroscopic continuous space at Planck's length is its diminutive size and the resulting lack of. experimental information that could guide our theoretical speculations. Instead, the available experimental input is located in the particle sector, at distance scales that are generally deemed irrelevant for the idea. Indeed, with field-theoretical approaches describing the behaviour of elementary particles fairly well, the idea to link the particle sector with the concept of the emergence of space may appear far-fetched. Yet-although something may indeed happen at the Planck distance scale-important information on the idea of space emergence may still reside at the particle and hadronic scales. After all, with space regarded as an attribute (or derivative) of matter, and with the discretization of mass hinting at some form of the discretization of space, it is the variety of particles and the spectrum of their masses that should direct our ideas on the quantization of space.

\section{Mass and Distance Scales}

\subsection{Planck Scale}

The lack of experimental input that could direct theoretical research on quantum gravity raises various questions. In particular, one may doubt both the need for the existence of the underlying quantized space and the relevance of Planck scale to such ideas. Indeed, gravity may be a strictly classical long-distance (residual?) phenomenon that does not have an underlying quantum counterpart (Wüthrich 2005). Furthermore, as observed by some authors (Amelino-Camelia; Baez 2001) and as clearly voiced by Meschini (2007), the only links between the general idea of an underlying quantized space and the Planck natural units are the dimensional analysis and the theoretical ad hoc considerations of Planck-size black holes. However, as pointed out in Meschini (2007), dimensional analysis is not a trustworthy tool that could provide us with reliable information on the realm of the unknown. This claim is justified in Meschini (2007) with a few examples from the history of physics which show that dimensional analysis becomes reliable only when the underlying theory is already known. For example, Meschini considers estimates of the size of hydrogen's atom and its binding energy that could have been made on the basis of dimensional analysis before any knowledge of Planck constant and the associated quantum features of reality were available. Assuming that hydrogen atom is built from electron and proton and accepting that the relevant dimensional constants are those involved in the classical theories of mechanics and electromagnetism, he shows that the predicted numbers are some five orders of magnitude away from their physical values.

On the other hand, the field-theoretical approach of the Standard Model works quite well down to the distances of the order of some $10^{-16} \mathrm{~cm}$, three orders of magnitude below typical hadronic size. On this basis one may hope that no change in the properties of space 
should occur for still smaller distances and that this trend continues down to the naturally distinguished scale of Planck's length. Yet, such a point of view ignores the fact that our conclusions concerning properties of space at small distances do not follow from direct observations but are inferred with the help of quantum field theory (which itself has a hybrid classical-quantum nature (Finkelstein 1969, 1972). In particular, it also ignores the issue of quantum nonlocality which questions the classical conception of an underlying spacetime even at macroscopic distances. Of course, quantum nonlocality does not directly jeopardize relativistic local quantum field theory. Yet, there is a clear tension between the classical and quantum descriptions, between locality and nonlocality. The deep and unaswered question is how classical locality emerges out of quantum nonlocality.

Now, experiments show that nonlocal quantum correlations appear at all probed distance scales. It looks as if spacial distances were completely irrelevant at the 'true' quantum level. It seems that the macroscopic spacetime of the classical description of nature emerges from the underlying quantum layer in a subtle and intrinsically nonlocal (holographic?) way, as the recent idea of building spacetime from quantum entanglement also suggests. There does not seem to be a reason to choose the Planck distance scale over the particle/hadronic scale (or the universe scale) as more appropriate for the emergence of the classical spacetime. From the vantage point of quantum nonlocality the Planck distance scale seems to lose its priviliged status. In fact, it was argued in the quantum gravity context (Bojowald 2007) that the typical distance scale $l_{Q S}$ relevant for the idea of space quantization may be dynamical and much larger than $l_{P}: l_{P}<<l_{Q S}<<l_{U}=c H_{0}^{-1}$ (where $l_{U}$ is the observable radius of the Universe and $H_{0}$ is the Hubble constant), and that the corresponding mass scale $m_{Q S}=h /\left(c l_{Q S}\right)$ is much smaller than $m_{P}$.

\subsection{Hadronic Scale}

The case for space quantization and emergence may look altogether different when other fundamental constants are admitted into the game. For example, there seems to be no reason why cosmological constant $\Lambda=1.19 \times 10^{-56} \mathrm{~cm}^{-2}\left(\approx\left(1 / l_{U}\right)^{2}\right)$ should not be regarded as a constant as important as $h, G$, and $c$. If $\Lambda$ is relevant, one can form (after Wesson (2004)) two additional mass scales that differ from $m_{P}$, namely

$$
m_{W}=(h / c) \sqrt{\Lambda / 3}=1.39 \times 10^{-65} \mathrm{~g},
$$

and

$$
m_{U}=\left(c^{2} / G\right) \sqrt{3 / \Lambda}=2.14 \times 10^{56} \mathrm{~g},
$$

with the Planck mass being their geometrical mean

$$
m_{P}^{2}=m_{W} m_{U} .
$$

Masses $m_{U}$ and $m_{W}$ are connected by an enormous dimensionless quantity:

$$
N=m_{U} / m_{W}=3 c^{3} /(h G \Lambda)=15.4 \times 10^{120}=m_{U}^{2} / m_{P}^{2} .
$$

The Wesson mass $m_{W}$, being proportional to $h$ and independent of $G$, may be interpreted as the quantum of mass. The other mass, $m_{U}$, which is independent of $h$, and thus seems appropriate for the classical limit, is of the order of the mass of the observable Universe. Now, with Planck mass being around one tenth of the mass of a flea, one may convincingly argue that $m_{P}$ corresponds to the classical and not the quantum realm. What meaning should be assigned then to Planck length which is directly related to $m_{P}$ by standard quantum considerations: $l_{P}=h /\left(m_{P} c\right)$ ? Does it make sense to apply 
quantum ideas to flea-weight objects in this way? Why is the interest of the majority focused on space and tiny distances $\left(l_{P}\right)$, and not on matter and tiny masses (e.g. $\left.m_{W}\right)$ ? Shouldn't the length and mass scales $l_{Q S}$ and $m_{Q S}$ be both far from the classical realm?

It may be observed that mass scales $m_{W}, m_{P}$, and $m_{U}$ are defined by expressions in which one of the four fundamental constants is missing (respectively: $G, \Lambda$, and $h$ ) (Burikham et al. 2016). Thus, one additional mass scale (the one without $c$ ) may be singled out as potentially important. It is

$$
m_{N}=m_{W} N^{1 / 3}=m_{U} N^{-2 / 3}=\left(\left(h^{2} / G\right) \sqrt{\Lambda / 3}\right)^{1 / 3}=0.346 \times 10^{-24} \mathrm{~g} .
$$

This number, which is smaller than the Planck mass by some 20 orders of magnitude, i.e.

$$
m_{P}=m_{N} N^{1 / 6},
$$

should be compared with the electron, pion and nucleon masses: $m_{e}=0.91 \times 10^{-27} \mathrm{~g}$, $m_{\pi}=0.25 \times 10^{-24} \mathrm{~g}, m_{n}=1.67 \times 10^{-24} \mathrm{~g}$. The fact that $m_{N}$, the particular combination of $h, G$, and $\Lambda$, is of the order of $m_{n}$ (or $m_{\pi}$ ) was noticed by many authors and is sometimes called the Eddington-Weinberg relation (Eddington 1946; Weinberg 1972) (see also Burikham et al. (2016) and Funkhouser (2008)). Furthermore, the distance scale related to $m_{N}$ by typical quantum considerations, ie. $l_{N}=h /\left(m_{N} c\right)=6.37 \times 10^{-13} \mathrm{~cm}$, is obviously of the order of typical hadronic size. Note that, unlike $l_{P}$ and $m_{P}$, both the distance and mass scales $l_{N}$ and $m_{N}$ are relatively small with respect to typical classical macroscopic distances and masses. Are hadronic scales relevant for the idea of space quantization?

In fact, there is not one but two a priori independent mass-related parameters that describe the hadronic spectrum. The first is the mass of the lowest lying hadronic states such as nucleon that fits the Eddington-Weinberg relation. The other parameter is concerned with the pattern of excited hadronic states. Here, the relevant feature of the hadronic spectrum is the appearance of linear Regge trajectories which describe infinite 'towers' of similar hadronic resonances (i.e. the recurrences of the ground state mesons and baryons with increasing spins $J$ and masses $m$ ) according to the generic formula:

$$
J=\alpha_{0}+\alpha^{\prime} m^{2}
$$

where $\alpha_{0}$ is the 'intercept' and $\alpha^{\prime}$-the slope of the trajectory. The slopes of all trajectories are similar: $\alpha^{\prime} \approx 0.9 \mathrm{GeV}^{-2}$ (with masses measured in GeV's, the units of energy). For example, for nucleon one has $1 / 2=\alpha_{0, n}+\alpha^{\prime} m_{n}^{2}$ (i.e. the intercept of nucleon trajectory is $\alpha_{0, n} \approx-1 / 2$ ). The recurrences of nucleon appear at $J=1 / 2,5 / 2,9 / 2,13 / 2, \ldots$ and their masses $m$ are given by Regge formula (7). Quark confinement ensures that such hadronic towers are infinite. When proper care is taken of spin dimension the universal slope is equal to

$$
\alpha^{\prime}=h /\left(2 \pi \Delta m^{2}\right) \approx 0.378 \times 10^{21} \mathrm{~cm}^{2} /(\mathrm{g} \mathrm{s})
$$

(experiment tells us that $\Delta m^{2}$, which describes the spacing of the masses of Regge recurrences, is approximately equal to proton mass squared). The slope $\alpha^{\prime}$ takes care of the string-like character of excited hadrons (the emergence of interquark strings) and permits one to express momenta as proportional (not inversely proportional) to positions via the dimensional constant

$$
\kappa_{R}=c^{2} / \alpha^{\prime}=2.37 \mathrm{~g} / \mathrm{s},
$$

which describes string tension and has the dimension of momentum/position (ie. $(\mathrm{g} \mathrm{cm} / \mathrm{s}) /$ $\mathrm{cm})$. 
It should be clear that the mass scale of ground state hadrons (which is of the order of $10^{-24} \mathrm{~g}$ ), and the slope of Regge trajectories (with $\kappa_{R}$ of the order of a few g/s) are two a priori independent parameters. After all, with $m_{n}\left(\right.$ or $\left.m_{\pi}\right)$ fixed, the relevant Regge trajectories could still be very steep or very flat (with $\Delta m^{2}<<m_{n}^{2}$ or $\Delta m^{2}>>m_{n}^{2}$ ). Yet the Regge scale of Eq. (9) satisfies a relation somewhat similar to the Eddington-Weinberg relation of Eq. (5). Indeed, we observe that from the four fundamental constants $h, G, c$, and $\Lambda$ one can form two constants of dimension $g / s$ :

the 'classical' constant

$$
\kappa_{C}=c^{3} / G=4.04 \times 10^{38} \mathrm{~g} / \mathrm{s},
$$

and the 'quantum' constant

$$
\kappa_{Q}=h \Lambda=0.79 \times 10^{-82} \mathrm{~g} / \mathrm{s} .
$$

which differ by the $N$ factor of Eq. (4). From Eq. (9) one finds that

$$
\kappa_{R}=5.9 \times 10^{-39} \kappa_{C},
$$

with the proportionality factor not far from $N^{-1 / 3}=0.4 \times 10^{-40}$. Thus, up to a factor of 100 , one has

$$
\kappa_{R} \approx N^{-1 / 3} \kappa_{C}=c^{2}\left(\frac{h \Lambda}{3 G^{2}}\right)^{1 / 3},
$$

which gives a rough estimate of Regge slope:

$$
\alpha^{\prime} \approx\left(\frac{3 G^{2}}{h \Lambda}\right)^{1 / 3}=55 \times 10^{21} \mathrm{~cm}^{2} /(\mathrm{g} \mathrm{s}) .
$$

The presence of a factor close to $N^{-1 / 3}$ in expression (12) constitutes a large number coincidence that seems to have not been noticed earlier. The fact that the particular combination of $h, G$, and $\Lambda$ given in Eq. (14) is of the order of $\alpha^{\prime}$ may be viewed as a counterpart of the Eddington-Weinberg relation of Eq. (5). Yet, it concerns not just the nucleon, but the whole Regge tower (trajectory) of hadronic resonances. The appearance of the same factor of $N^{ \pm 1 / 3}$ in connection with hadronic parameters $m_{n}$ and $\alpha^{\prime}$ points to their common origin.

With $m_{n}$ and $l_{n}$ being both much smaller than the macroscopic masses and distances it was contemplated quite early that the spacetime-based description of nature breaks down at the distance scale of hadronic physics (Zimmerman 1962) ${ }^{1}$. A decade or more after the introduction of quarks this idea was still on the mind of several physicists. For example, Penrose viewed his spin-network-induced twistor conceptions on the emergence of spacetime as appropriate at the hadronic scale (Penrose 1974). Today we know that hadrons are made of quarks which are conceived as pointlike objects. This discovery is usually taken to mean that there is no close connection between hadronic physics and the idea of the emergence of spacetime. Yet, one may argue that such a conclusion is premature as it is based

\footnotetext{
1 Strictly speaking, the classical notions of space and time are valid on the macroscopic level only, or (as put in Zimmerman (1962)): 'in situations where (...) a dense assembly of clocks and rods may be introduced without significant alteration of the physical situation'. Thus, the application of the classical picture of spacetime to hadron-level physics already constitutes a far-reaching extrapolation. It is achieved by way of merging the classical and quantum aspects of reality in a hybrid classical-quantum approach known under the name of quantum field theory (Finkelstein 1969, 1972).
} 
on a misidentification of current (admittedly very successful) field-theoretical description of strong interactions with physical reality. One may believe that the current SM description constitutes an idealization that will yield in time to a deeper description of elementary particles, a description more closely associated with the nature of spacetime (Penrose 1968). The search for physics 'beyond the Standard Model' does not have to mean 'at smaller distances' or 'at larger energies'. It may mean 'beyond the field-theoretical framework', or 'beyond the current conception of spacetime'.

\section{Internal Quantum Numbers and Phase Space}

\subsection{Nonrelativistic Phase Space}

The Standard Model contains several ingredients that are put in by hand. We do not know why there are three generations of fundamental fermions or why each generation is composed of two leptons and two sets of three quarks, all these particles being characterized by such internal quantum numbers as weak isospin, hypercharge, and color. It seems that the first problem that should be addressed is the issue of this generation structure.

With space viewed as an attribute of matter, properties of macroscopic 3D space and time should be associated with the properties of elementary particles. Indeed, the quantum numbers of spin and parity are connected with spatial 3D rotations and reflections, while the existence of particles and antiparticles may be linked to the operation of time reversal. With spatial quantum numbers being associated with the nonrelativistic conception of space and time, it is natural to expect that their existence could be deduced via some appropriate nonrelativistic analysis. ${ }^{2}$ Indeed, although inclusion of relativity leads to left and right spinors transforming differently under Lorentzian boosts, it does not affect the very existence and nonrelativistic properties of such concepts as spin or parity. At this point it is interesting to note that the above argument concerning the relative unimportance of special relativity in the study of some aspects of particle physics seems to go hand in hand with the absence of $\mathrm{c}$ in the expression for $m_{N}$, which is essentially the hadronic scale.

Given the existence of the nonrelativistic connection between space and spatial quantum numbers, it is tempting to expect that the internal quantum numbers could be associated with some nonrelativistic extension of the 3D space + time picture. Although relativity has to appear at some later stage of any full discussion, its introduction should not affect the main ('nonrelativistic') conclusions of such an approach. Furthermore, we think that it is too early for the inclusion of special relativity at the level of individual colored quarks. Indeed, we do not understand some important aspects of intra-hadronic physics already at the nonrelativistic level (for more details see Sect. 4.3). Thus, for our purposes, we may restrict attention to a nonrelativistic case. Now, the existence of dimensional constant $\kappa_{R}$ permits expressing positions as proportional to momenta, and allows the introduction of additional symmetry between these two sets of coordinates of the $6 \mathrm{D}$ phase space. ${ }^{3}$ With $\kappa$ having dimension [momentum/position], such a symmetry does not have much to do with the quantum connection which involves Planck constant of dimension [momentum $\times$ position].

\footnotetext{
${ }^{2}$ Although the existence of particles and antiparticles is generally viewed as an implication of the relativistic Dirac approach, it may be inferred from nonrelativistic analysis as well (Horzela and Kapuścik 2003).

${ }^{3}$ For example, the existence of $\kappa$ permits a replacement of positions by momenta and vice versa (actually $\mathbf{x} \rightarrow \mathbf{p}, \mathbf{p} \rightarrow-\mathbf{x})$, a 'reciprocity' symmetry originally introduced by Born (1949) in connection with the problem of mass.
} 
Such a phase-space-based description of reality seems to constitute a very natural and truly minimal 'extension' of the standard 3D description. Indeed, it may be viewed as (in a sense) 'a null extension', for it does not introduce any additional dimensions of (position) space. It is therefore more minimal than, for example, the approach of Trayling and Baylis (2001) which assumes seven spatial dimensions. ${ }^{4}$ In addition, the phase space approach realizes the philosophical condition of a symmetric treatment of things and processes (i.e. positions of things and their changes or, in other words, motions) as advocated by such thinkers as Heraclitus, Leibniz, and—most notably_Whitehead (Irvine 2010; Kraus 1979; Żenczykowski 2014). The utmost parsimony of this extension and its deep philosophical underpinning makes one hope that the expected additional phase-space-related quantum numbers could be identified with some observed internal quantum numbers. Of course, the above arguments should be backed by other arguments, ideally of observational or experimental nature. Such arguments will be provided later on.

\subsection{Linearization}

The simplest argument that leads from the level of macroscopic classical space (of either positions $\mathbf{x}$ or momenta $\mathbf{p}$ ) to the level of spatial quantum numbers is supplied by the Dirac linearization idea. According to this idea the 3D rotational invariant of momentum square $\mathbf{p}^{2}$ may be written as a product of two identical factors $\mathbf{A} \cdot \mathbf{p} \equiv \sum_{m} A_{m} p_{m}$, linear in momentum p:

$$
\mathbf{p}^{2}=(\mathbf{A} \cdot \mathbf{p})(\mathbf{A} \cdot \mathbf{p})
$$

where $\mathbf{A}$ is some momentum-independent object that behaves like $\mathbf{p}$ under rotations (so that $\mathbf{A} \cdot \mathbf{p}$ is a rotational invariant) and satisfies certain requirements that follow from (15). Specifically, as terms proportional to $p_{m} p_{n}$ are absent on the l.h.s. above, the $A_{m}$ 's must satisfy anticommutation rules

$$
A_{m} A_{n}+A_{n} A_{m}=2 \delta_{m n}
$$

which means that $A_{m}$ cannot be represented by ordinary numbers. As is well known, the above equation may be satisfied if one takes $A_{m}=\sigma_{m}$ where $\sigma_{m}$ are Pauli $(2 \times 2)$ matrices. Thus, linearization connects the rotational properties of the classical 3D macroscopic space with the quantum concept of $\operatorname{spin} S_{k}=\sigma_{k} / 2$.

When an extension from the 3D momentum (or position) space to the $6 \mathrm{D}$ phase space is investigated, it is natural to consider ${ }^{5}$ the phase-space analogue of $\mathbf{p}^{2}$, i.e. $\mathbf{p}^{2}+\mathbf{x}^{2}$ and to attempt its linearization à la Eq. (15):

$$
\mathbf{p}^{2}+\mathbf{x}^{2}=(\mathbf{A} \cdot \mathbf{p}+\mathbf{B} \cdot \mathbf{x})(\mathbf{A} \cdot \mathbf{p}+\mathbf{B} \cdot \mathbf{x})
$$

where $A_{m}$ and $B_{n}$ are the analogues of $A_{m}$ in Eq. (15). Let us now look at some details this idea entails. With momentum and position coordinates considered as classical (i.e. commuting) variables, the above equality requires six objects $A_{m}$ and $B_{n}$ to satisfy

\footnotetext{
${ }^{4}$ In paper of Trayling and Baylis (2001) the first three dimensions are the (observed) dimensions of position space, while the remaining four are added to make possible such an enlargement of the underlying geometric algebra that would permit - with the help of the concept of an algebraic spinor - the incorporation of all eight fermions of one generation of the Standard Model.

${ }^{5}$ For simplicity in the following we measure $\mathbf{p}$ and $\mathbf{x}$ in such units that both $\kappa$ and $h$ are 1 .
} 
anticommutation conditions analogous to (16), which define the Clifford algebra of 6D phase space. One finds that these conditions may be satisfied with $A_{m}$ and $B_{n}$ represented by $8 \times 8$ matrices (Żenczykowski 2007, 2008, 2014, 2015). A convenient representation of these six elements is provided by tensor products of Pauli matrices:

$$
\begin{gathered}
A_{m}=\sigma_{m} \otimes \sigma_{0} \otimes \sigma_{1}, \\
B_{n}=\sigma_{0} \otimes \sigma_{n} \otimes \sigma_{2} .
\end{gathered}
$$

When momenta and positions are considered as quantum variables, then - due to the nonzero value of commutator $\left[x_{m}, p_{n}\right]=i \delta_{m n}$-there appears an additional term on the 1.h.s. of (17), i.e. one gets

$$
(\mathbf{A} \cdot \mathbf{p}+\mathbf{B} \cdot \mathbf{x})(\mathbf{A} \cdot \mathbf{p}+\mathbf{B} \cdot \mathbf{x})=\mathbf{p}^{2}+\mathbf{x}^{2}+R
$$

with

$$
R=-\frac{i}{2} \sum_{k}\left[A_{k}, B_{k}\right]=\sum_{k} \sigma_{k} \otimes \sigma_{k} \otimes \sigma_{3}
$$

After introducing the element

$$
B=i A_{1} A_{2} A_{3} B_{1} B_{2} B_{3}=\sigma_{0} \otimes \sigma_{0} \otimes \sigma_{3},
$$

multiplying a rescaled version of Eq. (19) by $B$ :

$$
Q \equiv \frac{1}{6}\left[\left(\mathbf{p}^{2}+\mathbf{x}^{2}\right)_{v a c}+R\right] B
$$

(where $\left(\mathbf{p}^{2}+\mathbf{x}^{2}\right)_{\text {vac }}=3$ is the lowest eigenvalue of $\mathbf{p}^{2}+\mathbf{x}^{2}$ ), and defining matrices

$$
\begin{gathered}
I_{3}=\frac{1}{2} B, \\
Y=\frac{1}{3} R B=\frac{1}{3} \sum_{k} \sigma_{k} \otimes \sigma_{k} \otimes \sigma_{0},
\end{gathered}
$$

one obtains

$$
Q=I_{3}+Y / 2 \text {. }
$$

It may be checked that the $8 \times 8$ matrices $I_{3}$ and $Y$ commute among themselves and are invariant under ordinary $3 \mathrm{D}$ rotations and reflections. Consequently, their eigenvalues constitute natural candidates for internal quantum numbers. With these eigenvalues being

$$
\begin{array}{ll}
I_{3} \rightarrow \pm 1 / 2, & \text { (for any } Y \text { ), } \\
Y \rightarrow-1,+1 / 3,+1 / 3,+1 / 3, & \text { (for any } I_{3} \text { ), }
\end{array}
$$

the eigenvalues of $Q$ are $(0,+2 / 3,+2 / 3,+2 / 3,-1,-1 / 3,-1 / 3,-1 / 3)$, identical with the charges of eight fundamental fermions composing a single generation of the SM. It is therefore natural to identify (25) with the Gell-Mann-Nishijima formula for electric charge $Q$, and $I_{3}$ and $Y$ with weak isospin and hypercharge. 
Table 1 Decomposition of hypercharge eigenvalues

\begin{tabular}{lllll}
\hline$Y_{1}$ & $Y_{2}$ & $Y_{3}$ & $Y$ & Particle \\
\hline$-1 / 3$ & $+1 / 3$ & $+1 / 3$ & $+1 / 3$ & Red quark \\
$+1 / 3$ & $-1 / 3$ & $+1 / 3$ & $+1 / 3$ & Blue quark \\
$+1 / 3$ & $+1 / 3$ & $-1 / 3$ & $+1 / 3$ & Green quark \\
$-1 / 3$ & $-1 / 3$ & $-1 / 3$ & -1 & Lepton \\
\hline
\end{tabular}

\subsection{Rishons or 'Partial Hypercharges'}

The triple appearance of the $Y$ eigenvalue of $+1 / 3$ is naturally associated with the triplicity of the color quantum number. It is instructive to see how this triplicity emerges. From (19) we observe that the hypercharge $Y$ is built as a sum of three mutually commuting 'partial hypercharges'

$$
Y_{k}=-\frac{i}{6}\left[A_{k}, B_{k}\right] B=\frac{1}{3} \sigma_{k} \otimes \sigma_{k} \otimes \sigma_{0} .
$$

Calculation shows that the values of $Y$ are constructed from $Y_{k}$ in the way indicated in Table 1. The rightmost column in Table 1 provides the correspondence between the structure of $Y$ and the quartet composed of a lepton and three colored quarks. The triplicity of color is attributed here to three different orderings in which $Y=+1 / 3$ may be built out of $-1 / 3,+1 / 3$, and $+1 / 3$.

It appears (Żenczykowski 2015) that the pattern exhibited in Table 1 is in one-to-one correspondence with the way in which the charges of leptons and quarks are built out of the charges of their alleged subparticles, the so-called 'rishons' of the Harari-Shupe (HS) model (Harari 1979; Shupe 1979). In that model the eight fermions of a single SM generation are conceived as ordered triplets of two 'truly fundamental' spin-1/2 subparticles (rishons) $T$ and $V$ of charges $+1 / 3$ and 0 respectively. For example, the red $u$ quark is identified with the triplet $V T T$, while the neutrino $v$-with $V V V$. With only two types of rishons the HS scheme is very economic in the number of fundamental particles. Yet, it exhibits many shortcomings in other places. In particular, it predicts the existence of unobserved particles (e.g. spin-3/2 partners of leptons and quarks), its concept of color is not connected with the $S U(3)$ color group of the SM, it violates the condition of fermion antisymmetrization at the rishon level, etc., etc. It turns out that in the phase-space framework these shortcomings of the HS rishon model do not appear. The basic reason is that in that approach the partial hypercharges (the counterparts of HS rishons) constitute algebraic components of the hypercharge operator only. They do not reside on any subparticles. Lepton and quarks of a single SM generation are connected by phase-space-induced symmetry, but its explanation in terms of subparticles is neither needed nor possible. For a more detailed presentation of the relevant arguments see Żenczykowski (2016). By Ockham's razor, the phase-space explanation of the observed pattern of fundamental fermions is vastly superior to that provided by the original rishon model.

The shortcomings of the HS model result from the intuitive wish to divide matter again and again. Yet, the divisibility of matter must come to an end. As noted by Heisenberg (1976), this comes about as a change in the meaning of the word 'to divide' so that after several steps down the ladder of compositeness the concept of further subdivision loses most of its original meaning. One of the lowest and most important steps of this ladder seems to occur when the concept of macroscopic separability is 
lost, i.e. during the transition from the hadronic to the quark level. As the phase-space scheme suggests, going further down (to the supposed rishon level) seems to require a strictly algebraic understanding of the compositeness of matter (i.e. the construction of hypercharge from 'partial hypercharges' that do not reside on subparticles) and, consequently, the inapplicability of the ordinary conception of matter below the lepton/ quark level. The conceptual superiority of the phase-space scheme over that provided by the original HS model strongly suggests that the ordinary conception of matter starts at the lepton/quark scale, not below it. With space understood as an attribute of matter, it seems therefore that it is the transition from the partial hypercharge ('rishon') level through the lepton/quark level and on to the hadronic level (i.e. the emergence of matter) that should be relevant for the understanding of the emergence of space. The Planck scales seem quite irrelevant here.

\section{Generalization of Mass, Emergence of Hadrons}

\subsection{Problem of Mass}

The problem of mass is a thorny one. For a long time the main controversy seemed to have been between the view that mass is an intrinsic property of a given particle and the opinion that it results from the interaction of that particle with other objects. According to Wigner (1939), from the group-theoretical standpoint the observed particles may be labelled by two space-related properties, namely spin and mass, associated with rotations and translations respectively. This seems to suggest similar physical origins of mass and spin. Yet, the current view treats spin and mass somewhat asymmetrically: it holds that while spin is an intrinsic property of a particle, it is the interaction with the Higgs field that generates the masses of originally massless particles.

Still, the Higgs mechanism (as it stands now) does not provide us with a substantial improvement in our understanding of the problem of mass. As noted in Hansson $(2014 \mathrm{a}, \mathrm{b})$, it 'merely replaces one set of unknown parameters (particle masses) with an equally unknown set of parameters (coupling constants to the Higgs field(s)), so nothing is gained in the fundamental understanding of masses.' The problem of mass is further exacerbated by the existence of mixing between different generations. Thus, we need a principle deeper than the Higgs mechanism. It should provide a single rationale behind the existence of the whole variety of fundamental particles, explain the observed pattern of their masses and mixings, and reduce the number of free SM parameters. For example, Hansson $(2014 a$, b) argues that the relative values of neutrino, electron, and quark masses are correlated with the strength of fundamental interactions in which these particle participate. Given our ignorance as far as origin of mass is concerned it is obvious that one should welcome any additional light that could be shed on this problem.

\subsection{Phase Space and Mass}

The phase-space scheme provides an attractive explanation of the origin of several internal quantum numbers. It turns out that it has other interesting implications as well. They bear on the idea of space as a property of matter. Generalization of the concept of mass is one 
of them. It is this generalization that (as we believe) may help us in future to uncover how classical macroscopic space emerges from (and is constructed as a limiting property of) the underlying material quantum layer. It was observed by Born (1949) that the ordinary concept of mass distinguishes between momentum and position variables. Indeed, mass of individual free physical bodies always enters into dispersion formulas in association with momentum (and not with position), be it in a nonrelativistic or relativistic expressions for particle energies i.e.

$$
E=\mathbf{p}^{2} / 2 m \text { or } E^{2}=\mathbf{p}^{2}+m^{2} .
$$

Born's reciprocity symmetry between $\mathbf{p}$ and $\mathbf{x}$ violates this association of mass with momentum.

The introduction of $\kappa$ permits a completely parallel treatment of momentum and position variables and suggests the introduction of phase-space invariant $\mathbf{p}^{2}+\mathbf{x}^{2}$. This invariant admits the consideration of transformations that replace momenta with positions in various ways, not only via Born's reciprocity transformation. Specifically, consider the following rotations in phase space:

$$
\begin{aligned}
p_{1}^{\prime} & =p_{1} \cos \phi+x_{3} \sin \phi, \\
x_{3}^{\prime} & =x_{3} \cos \phi-p_{1} \sin \phi, \\
p_{2}^{\prime} & =p_{2}, \\
x_{2}^{\prime} & =x_{2}, \\
p_{3}^{\prime} & =p_{3} \cos \phi-x_{1} \sin \phi, \\
x_{1}^{\prime} & =x_{1} \cos \phi+p_{3} \sin \phi .
\end{aligned}
$$

Invariance of $\mathbf{A} \cdot \mathbf{p}+\mathbf{B} \cdot \mathbf{x}$ requires that $A_{m}$ and $B_{n}$ transform as above with $p_{m} \rightarrow A_{m}$ and $x_{n} \rightarrow B_{n}$, and with similar replacements for primed objects. For $\phi=\pi / 2$ one then immediately finds that

$$
\begin{aligned}
& A_{1}^{\prime}=B_{3}, \quad A_{2}^{\prime}=A_{2}, \quad A_{3}^{\prime}=-B_{1}, \\
& B_{3}^{\prime}=-A_{1}, \quad B_{2}^{\prime}=B_{2}, \quad B_{1}^{\prime}=A_{3},
\end{aligned}
$$

and, consequently,

$$
\begin{aligned}
& B^{\prime}=B, \\
& Y_{1}^{\prime}=-Y_{3}, \\
& Y_{2}^{\prime}=Y_{2}, \\
& Y_{3}^{\prime}=-Y_{1},
\end{aligned}
$$

i.e. nothing happens to the values of $I_{3}=B / 2$ and $Y_{2}$, while the values of $Y_{1}$ and $Y_{3}$ are interchanged (with additional negative signs). This leads to Table 1 being transformed (row by row) into Table 2 . We see that the lepton and the blue quark are interchanged, while the red and green quarks are unaffected. One may say that a quark of a given color is a lepton appropriately rotated in phase space.

Consider now the effect that this transformation has on the connection between phase space variables and the classical concept of mass. Let us focus on the standard dispersion 
Table 2 Structure of $Y$ after rotation in phase space

\begin{tabular}{lllll}
\hline$Y_{1}^{\prime}$ & $Y_{2}^{\prime}$ & $Y_{3}^{\prime}$ & $Y^{\prime}$ & Particle \\
\hline$-1 / 3$ & $+1 / 3$ & $+1 / 3$ & $+1 / 3$ & Red quark \\
$-1 / 3$ & $-1 / 3$ & $-1 / 3$ & -1 & Lepton \\
$+1 / 3$ & $+1 / 3$ & $-1 / 3$ & $+1 / 3$ & Green quark \\
$+1 / 3$ & $-1 / 3$ & $+1 / 3$ & $+1 / 3$ & Blue quark \\
\hline
\end{tabular}

relation for a macroscopic body, that is naturally extrapolated to free microscopic objects, such as a lepton. Then, according to Eq. (29), in the dispersion formula appropriate for the blue quark, the ordinary three-momentum $\left(p_{1}, p_{2}, p_{3}\right)$ is replaced by a 'mixed' triplet of phase space variables, i.e. by a new canonical momentum

$$
\left(p_{1}^{\prime}, p_{2}^{\prime}, p_{3}^{\prime}\right)=\left(x_{3}, p_{2},-x_{1}\right) .
$$

Thus, a free blue quark would have to satisfy a symmetry-related phase-space counterpart of Eq.(28) i.e. (for an originally relativistic relation)

$$
E^{2}=x_{3}^{2}+p_{2}^{2}+x_{1}^{2}+m^{2} .
$$

By analogy, similar mixed triplets and dispersion formulas (with cyclic relabelling $2 \rightarrow 3 \rightarrow 1 \rightarrow 2$ ) appear for the red and green quarks.

It may be objected that these implications of the phase-space scheme are totally unacceptable as they violate rotational, translational, and relativistic invariances of the surrounding macroscopic world. We think, however, that the appearance of such violations is not a vice but one of the greatest assets of the proposed scheme. In our view, such wouldbe violations lie at the origin of quark confinement (or-more conservatively speaking - of a novel perspective on it). In other words, we believe that it is due to the would-be violation of rotational and translational invariances by the quark dispersion formula that a single quark cannot be observed in the familiar classical 3D world in which these invariances obviously have to hold. Thus, unobservability of individual quarks is predicted by the scheme.

The proposed explanation of quark confinement seems to be in a manifest contradiction with the generally accepted standard picture of quarks and their confinement via gluonmediated long-range QCD interactions. Therefore, a first reaction to the phase-space picture could be to discard it right away. Yet, before one makes such a hasty decision, one should discuss possible ways of resolving the conflict. In particular, one should first address such issues as the possible connection between phase-space ideas and the ordinary description of hadron substructure in terms of quantum quark fields, and the disagreement with the widely embraced standard view on quark masses. Then, one may turn to the discussion of a seemingly unacceptable description of quark confinement.

\subsection{Defense of Phase Space Picture}

First, let us stress that in our phase-space considerations we are concerned with the dispersion relation of a free quark (i.e. in the precise association of the concept of quark mass with phase-space variables), not with other quark properties such as e.g. quark spin, chiral properties, etc. which are assumed to be fully relevant for the field-theoretical description of the interaction of quarks with external probes such as photon or weak bosons. The 
non-standard dispersion relation that an individual quark is supposed to satisfy is viewed as a classical constraint that should be introduced into the quantum field-theoretical framework from the 'outside' (i.e. from the classical level, just as it is done in the case of the standard dispersion formula). The fact that in our approach the individual quarks are expected to subscribe to a non-standard dispersion relation does not mean that in the quantum field-theoretical approach (and from the point of view of the 3D space + time background and color-blind external probes) they cannot be described with the help of a pair of ordinary (left and right) spinorial fields (the bispinor $q(x)$ ), as it is standardly done in quark/hadron phenomenology and in QCD. A large part of standard phenomenological and field-theoretical description of hadrons in terms of quark substructure is thereby automatically accepted. What should be skipped are those parts of that picture which involve the classically-motivated on-mass-shell constraints such as e.g. the Dirac equation $(\not p-m) q(x)=0$ (Żenczykowski 2015). This is highly welcome as the use of ordinary on-mass-shell formulas for quarks is unacceptable on conceptual grounds: in the standard field-theoretical approach the ordinary on-mass-shell condition $\left(p^{2}=m^{2}\right)$ corresponds to spatial infinity which obviously cannot be reached by confined quarks. For similar reasons I find it hard to accept without reservations those quark-level calculations which involve standard quark propagators $1 /(\not p-m)$ : after all, the very idea of confinement forbids the existence of quark poles at $p^{2}=m^{2}$. In my view, such calculations have to be treated as approximations that may lead to a variety of artefacts and-for these reasons-cannot be really trusted. And indeed, not only are the standard quark propagators in conceptual conflict with the very unobservability of free quarks, but their carefree use does lead to incorrect predictions also in other, less obvious places (see eg. Żenczykowski 2006).

Second, when confronted with the above claim that the standard concept of mass is not appropriate for a quark, most physicists would presumably argue that our understanding of quark masses cannot be that bad: they would be inclined to belief in the soundness of standard quark mass extraction procedures. After all, respectable scientific literature accepts standard (Dirac) character of quark masses and lists quite precise values for these parameters. The point is, however, that - with free quarks being unobservable in asymptotic states - any extraction of quark mass from experimental data must depend heavily on theory, i.e. on the way quark 'mass' is defined and built into the relevant theoretical scheme. Obviously, there is no doubt that a quark may be assigned some effective mass parameter that can be extracted from hadronic level data. Yet, what is actually being extracted via the relevant theoretical procedures? Are the extracted quark masses the Dirac masses? Or are they mass-like parameters of a more general nature? Do we know the relevant theory sufficiently well for such extractions to be fully reliable? It turns out that the original, fifty years old prescription for the extraction of the so-called 'current' quark masses (via the 'current algebra' of hadronic currents) does not actually treat quarks as Dirac particles 'moving' within hadrons. ${ }^{6}$ For our purposes it is sufficient to say that in these extractions one uses global chiral properties of effective quark mass terms as well as spacetime concepts defined at the hadronic level. Some quark-level symmetries do enter into the game but no assumption is made concerning the existence of ordinary background space within hadrons. Moreover, in such extractions the on-mass-shell Dirac

\footnotetext{
${ }^{6}$ For a brief discussion of how the standard values of quark masses are extracted from experiment via 'current algebra' see eg. Żenczykowski (2015).
} 
condition $(\not p-m) q(x)=0$ does not have to be used (although it sometimes is), thus making it possible to avoid the pending conceptual conflict between its use and quark confinement.

More modern quark mass extractions are based on lattice QCD calculations in which, although the quarks are pictured on the background of classical spacetime, standard quark propagators do not appear. Thus, just as in the case of current algebra, there emerges no conceptual conflict with the unobservability of individual quarks in asymptotic states. The mass values extracted via such more sophisticated calculations are in good agreement with the old current algebra estimates. This seems to suggest that one may go beyond the simple current algebra picture and extend the standard spacetime concepts right into the hadronic 'interior'. Accordingly, the concept of quark mass may be regarded as quite standard, with its Dirac nature being masked by QCD confinement effects. Yet, we shall point out below that this extension seems to go too far.

Before we turn to the issue of the essential difference in the pictures of confinement, let us comment on some interesting aspects of the phase-space-induced view on the notion of mass that are missing in the SM approach. Namely, a study of all 64 elements of the Clifford algebra of phase space shows (Żenczykowski 2009, 2017, 2014) that there is only one element of this algebra (up to Born's reciprocity transformation) that may be associated with the concept of lepton mass. Its rotations in phase space / Clifford algebra (using Eq. (30) etc.) lead to three Clifford algebra elements that have to be associated with the concepts of mass for tri-colored quarks. It turns out that these elements are diagonal elements of a rank-2 tensor that is symmetric in $3 \mathrm{D}$ indices ${ }^{7}$, possibly hinting at the connection of quarks with the appearance of metric and thus with the idea of space emergence. Furthermore, the rotations in phase space involve string tension $\kappa_{R}$. This seems to indicate that the problem of mass does not really break up into two independent problems: the problem of the mass of fundamental fermions (Clifford algebra elements for lepton and quark masses) and the problem of the strength of interquark confining interactions (string tension, $\kappa_{R}$ ), as it is often thought nowadays. Instead, these two problems seem to constitute two closely related parts of a single puzzle.

Finally, we come to the third point-the issue of confinement and the difference between the phase-space view and the QCD picture. The conceptual simplicity and parsimony of the phase space approach strongly suggests that this approach contains several important elements of truth. If so, we should not reject it immediately but rather find a way to marry it with the QCD picture. This may be attempted if one accepts the view that both the phase-space ideas and the QCD picture are idealizations that may deviate from reality in various places. ${ }^{8}$ Thus, the phase-space and QCD pictures should be viewed as two different perspectives on the nature of quark confinement. As such they may benefit from each other. It is therefore gratifying that the two pictures exhibit important similarities. For example, the phase space approach provides the justification for the appearance of the $\mathrm{SU}(3)$ color symmetry group of QCD. Furthermore, both the phase-space picture and QCD

\footnotetext{
7 While Clifford algebras in general lead to antisymmetric tensors only, the phase-space-induced doubling of the 3D structure (the parallel treatment of position and momentum spaces) leads to elements that-from the point of view of our 3D world-are symmetric in 3D indices.

8 After all, all our theories are abstract descriptions, models built with the goal of representing various features of nature and applicable to its limited regions only (Heisenberg 1958). They must not be treated as the underlying and complete truths that are valid everywhere.
} 
involve the description of confinement in terms of strings, even though these strings are pictured differently.

Addressing the question of how to marry the phase-space view with the QCD picture requires going beyond the intended scope of the present paper. Nonetheless, we should mention here an idea that was put forward in Mulders $(2016,2018)$ and could be relevant to this question. The proposal of Mulders $(2016,2018)$ bears some resemblance to the phasespace scheme: it links the number of colors to the number of space dimensions, constructs leptons and quarks from one-dimensional structures somewhat akin to our interpretation of Harari-Shupe rishons, and does away right from the beginning with the confinement problem. At the same time it involves left and right objects, dynamical gluons, and more. An intriguing question is if such ideas can be adapted to our scheme, marrying it with QCD and bringing in a way to incorporate special relativity.

Now, while the phase-space picture (as it stands now) misses important elements of QCD (eg. gluons), QCD also seems to be deficient, although from a different angle. In particular, there are strong phenomenological indications from baryon spectroscopy that something important is missing in the original quark model/ QCD picture. It appears that the standard quark model and lattice QCD both predict the existence of many more excited baryonic states than experimentally observed (Capstick and Roberts 2000). More specifically, it seems that in excited baryons one internal spatial degree of freedom is frozen (this provides a direct suggestion that there is a close connection of quark-to-hadron transition, the mechanism of quark confinement, and hadronic scale with the idea of space emergence). On this issue Capstick and Roberts write (Capstick and Roberts 2000): 'If no new baryons are found, both QCD and the quark model will have made incorrect predictions, and it would be necessary to correct the misconceptions that led to these predictions. Current understanding of $Q C D$ would have to be modified and the dynamics within the quark model would have to be changed'. We conclude that it is not sufficient to calculate the masses of the ground-state mesons and baryons and to claim-on the basis of their approximate agreement with experimental values - that the relevant theoretical scheme is correct. One cannot view such successes of lattice QCD as a sufficient argument favoring the existence of standard spacetime background in hadronic 'interior'. The real challenge for the lattice QCD is the description of baryonic excited states. It is only after this is achieved that one can accept the applicability of unmodified QCD to the description of quark confinement. As the situation stands now, the apparent freezing of one spatial degree of freedom in excited baryons strongly suggests that the nature of hadronic 'interior' may be different from naive extrapolations. We repeat: the fact that one can describe quarks as quantum fields on the background of classical continuous spacetime and that many interesting conceptions (including various ideas on quark/hadron transition) were developed within such a picture does not mean that this background, assigned to the interior of hadrons by an extrapolation from the macroscopic domain, is fully adequate for a deeper description of quarks and hadronic structure.

Now, it is interesting to note that on the issue of hadronic 'interior' the phase-space picture seems to differ from standard quark approaches. The implication that masses of individual quarks should enter into rotationally and translationally non-invariant dispersion relations means that quarks cannot exist as individual free objects. It does not mean, however, that they cannot exist as inseparable components of multi-quark conglomerates provided these conglomerates satisfy all the invariances that ordinary objects are supposed to satisfy. The phase-space approach seems to admit the emergence of such conglomerates. Indeed, although in this approach a precise prescription for the construction of hadronic states is missing, an interesting argument that leads to the emergence of mesonic and 
baryonic structures may be given. This argument is based on an extension of the principle of additivity of physical momenta of ordinary macroscopically separable objects. According to this principle, the total momentum of a composite system of ordinary objects is given by the sum of the momenta of its components $\left(\mathbf{p}_{t o t}=\sum_{k} \mathbf{p}^{(k)}\right)$. In the phase space picture one may expect this trivial principle to be generalized to the principle of additivity of canonical momenta. This leads in particular to the addition of canonical momenta of three quarks of different colors, i.e. of

$$
\begin{gathered}
\left(p_{1}^{B}, p_{2}^{B}, p_{3}^{B}\right)=\left(-y_{1}, p_{2}, x_{3}\right), \\
\left(p_{1}^{R}, p_{2}^{R}, p_{3}^{R}\right)=\left(x_{1},-y_{2}, p_{3}\right), \\
\left(p_{1}^{G}, p_{2}^{G}, p_{3}^{G}\right)=\left(p_{1}, x_{2},-y_{3}\right),
\end{gathered}
$$

and suggests the appearance of a translationally invariant expression

$$
\left(p_{1}, p_{2}, p_{3}, x_{1}-y_{1}, x_{2}-y_{2}, x_{3}-y_{3}\right),
$$

which can be made rotationally covariant if the three quarks conspire so that $\mathbf{p}$ and $\mathbf{x}-\mathbf{y}$ are actually ordinary vectors. Thus, using the additivity principle one may construct expressions that satisfy the condition of proper rotational and translational behavior at the composite level, and are therefore appropriate for the description of ordinary objects. Accepting this generalization of the additivity principle leads to the emergence of expressions appropriate for the description of mesons and baryons, in a way somewhat similar to their standard group-theoretical description. Further discussion of this idea may be found in Żenczykowski $(2009,2014,2015,2017)$. Here, we would just like to point out that the additivity prescription treats the two a priori possible internal baryonic spatial degrees of freedom in an asymmetrical manner (as only one vector of internal displacement is present in Eq. (35)), which may have something to do with the apparent freezing of one internal degree of freedom in excited baryons. Thus, the phase space picture-if developed for the description of hadrons in an as yet unknown way- may provide hints on how the QCD picture should be modified. Obviously, with QCD requiring a prior acceptance of the existence of an underlying background spacetime, introduction of gluons into the phasespace approach should be postponed until a working idea on the emergence of spacetime is proposed.

\section{Conclusions}

In conclusion, I think that important information on the idea of space emergence could be extracted from the hadronic realm. There is a couple of arguments that support this point of view.

First, with space viewed as an attribute of matter, it seems that it is the discrete spectrum of masses of elementary particles that should define discrete properties of space in the microcosm. Thus, one should start not from discrete (or quantized) space but from discrete (or quantized) matter. Hadrons seem particularly relevant here as their spectrum comprises objects of all spins.

Second, the standard dimensional argument that singles out Planck mass scale (and the related distance scale) as relevant for space emergence has a natural counterpart that singles out the hadronic mass scale instead of Planck scale. Indeed, if one accepts four fundamental constants: $h, G, c$, and $\Lambda$, then the Planck mass scale $m_{P}$ is obtained if $\Lambda$ is not used, 
while the hadronic mass scale $m_{N}$ is obtained if $c$ is not used. As the hadronic mass scale $m_{N}$ is much farther from the classical realm than the Planck mass scale (which is essentially of classical size) it seems that it is $m_{N}$ (and not $m_{P}$ ) that should be relevant for the consideration of spacetime emergence. Moreover, in addition to $m_{N}$ there is another a priori independent scale (the Regge tension $\kappa_{R}$ ) that characterizes the hadronic spectrum and is far from the classical realm $\left(\kappa_{R}<<\kappa_{C}\right)$.

Third, the existence of hadronic spectrum parameter $\kappa_{R}$ supports a phase-space picture that provides a justification for the emergence of internal quantum numbers of weak isospin, hypercharge and color. As this picture is essentially unique in its parsimony, it is very attractive. It suggests an extension of the concept of mass (and a more general view on matter that involves a symmetric treatment of things and processes) and predicts the unobservability of individual quarks. It also seems to involve an asymmetric treatment of the two intra-baryonic spatial degrees of freedom, which are expected in simple extrapolations of the macroscopic conception of space to the hadronic 'interior'. Thus, it questions the idea of ordinary divisibility of space when going from the hadronic to the quark level, and suggests that standard ideas on intra-baryonic space constitute an approximation to reality only. Such a view on hadronic 'interior' is corroborated by phenomenological analyses of the spectrum of excited baryons which indicate that one internal spatial degree of freedom is frozen.

Fourth, extending the idea of the divisibility of matter below the lepton/quark level, as it is assumed in the rishon model, leads to many shortcomings which are automatically absent if one adopts a strictly algebraic interpretation of rishons as obtained in the phasespace picture.

To summarize, I think that the emergence of space may and should be viewed as a byproduct of the transition of matter from the (algebraic) rishon level via the (particle/ quasi-particle) lepton/quark level and on to the (particle) lepton/hadron level. The currently dominant view of continuous space emerging at the Planck distance scale seems to be in conflict with the philosophical position accepting logical priority of matter over space when this position is combined with the analysis of the salient properties of (quantized) matter.

Open Access This article is distributed under the terms of the Creative Commons Attribution 4.0 International License (http://creativecommons.org/licenses/by/4.0/), which permits unrestricted use, distribution, and reproduction in any medium, provided you give appropriate credit to the original author(s) and the source, provide a link to the Creative Commons license, and indicate if changes were made.

\section{References}

Amelino-Camelia, G. Quantum gravity phenomenology, physics/0311037.

Baez, J. C. (2001). Higher-dimensional algebra and Planck-scale physics. In C. Callender \& N. Huggett (Eds.), Physics meets philosophy at the Planck scale (pp. 177-195). Cambridge: Cambridge Univ. Press.

Bojowald, M. (2007). Quantum gravity and cosmological observations. AIP Conference Proceedings, 917, 130-137. arXiv: gr-qc/0701142.

Born, M. (1949). Reciprocity theory of elementary particles. Reviews of Modern Physics, 21, 463-473.

Burikham, P., Dhanawittayapol, R., \& Wuthicharn, T. (2016). A new mass scale, implications on black hole evaporation and holography. International Journal of Modern Physics A, 31(16), 1650089. arXiv: 1605.05866 .

Capstick, S., \& Roberts, W. (2000). Quark models of baryon masses and decays. Progress in Particle and Nuclear Physics, 45, S241-S331. 
Eddington, A. S. (1946). Fundamental theory. Cambridge: Cambridge University Press.

Finkelstein, D. R. (1969). Space-time code. Physical Review, 184, 1261-1271.

Finkelstein, D. R. (1972). Space-time code. 2. Physical Review D, 5, 320-328.

Funkhouser, S. (2008). A new large-number coincidence and a scaling law for the cosmological constant. Proceedings of the Royal Society of London A: Mathematical, Physical and Engineering Sciences, 464, 1345-1353. arXiv: physics/0611115.

Hansson, J. (2014). Physical origin of elementary particle masses. Electronic Journal of Theoretical Physics, 11, 87-100. arXiv: 1402.7033.

Hansson, J. (2014). On the origin of elementary particle masses. Progress in Physics, 10, 71-73. arXiv: 1211.3136.

Harari, H. (1979). A schematic model of quarks and leptons. Physics Letters B, 86, 83-86.

Heisenberg, W. (1958). Physics and philosophy: The revolution in modern science (p. 200). New York: Harper and Row.

Heisenberg, W. (1976). The nature of elementary particles. Physics Today, 29, 32-39.

Heisenberg, W. (1979). Ideas of the natural philosophy of ancient times in modern physics. In Philosophical problems of quantum physics. Woodbridge, CT: OxBow Press.

Horzela, A., \& Kapuścik, E. (2003). Galilean covariant Dirac equation. Electromagnetic Phenomena, 3 , 63-69.

Irvine, A. D. (2010). Alfred North Whitehead. In N. Edward Zalta (Ed.), The Stanford encyclopedia of philosophy, Winter. http://plato.stanford.edu/archives/win2010/entries/whitehead/.

Kraus, E. (1979). The metaphysics of experience: A companion to Whitehead's process and reality. New York: Fordham University Press.

Meschini, D. (2007). Planck-scale physics: Facts and beliefs, Foundations of Science, 12, 277-294. arXiv: gr-qc/0601097.

Mulders, P. (2016). The 3D structure of QCD and the roots of the standard model. EPJ Web of Conferences, $112,01014$.

Mulders, P. (2018). The 3D entangled structure of the proton; transverse degrees of freedom in QCD, momenta, spins and more. Few Body Systems, 59(2), 10. arXiv:1801.03664.

Penrose, R. (1968). Structure of spacetime. In C. M. DeWitt \& J. A. Wheeler (Eds.), Batelle Rencontres (pp. 121-235). New York: Benjamin.

Penrose, R. (1974). Twistors and particles: An outline. In Proceedings of the conference on quantum theory and the structures of time and space, Feldafing (pp. 129-145).

Shupe, M. (1979). A composite model of leptons and quarks. Physics Letters B, 86, 87-92.

Trayling, G., \& Baylis, W. (2001). A geometric basis for the standard-model gauge group. Journal of Physics A: Mathematical and General, 34, 3309-3324.

Weinberg, S. (1972). Gravitation and cosmology. New York: Wiley.

Wesson, P. S. (2004). Is mass quantized? Modern Physics Letters A, 19, 1995-2000. arXiv: gr-qc/0309100.

Wigner, E. P. (1939). On unitary representations of the inhomogeneous Lorentz group. Annals of Mathematics, 40, 149-204.

Wüthrich, C. (2005). To quantize or not to quantize: Fact and folklore in quantum gravity. Philosophy of Science, 72, 777-788.

Zimmerman, E. J. (1962). The macroscopic nature of space-time. American Journal of Physics, 30, 97-105.

Żenczykowski, P. (2006). Joint description of weak radiative and nonleptonic hyperon decays in broken SU(3). Physical Review D, 73, 076005.

Żenczykowski, P. (2007). Space, phase space and quantum numbers of elementary particles. Acta Physica Polon B, 38, 2053-2076.

Żenczykowski, P. (2008). The Harari-Shupe preon model and nonrelativistic quantum phase space. Physics Letters B, 660, 567-572.

Żenczykowski, P. (2009). Clifford algebra of nonrelativistic phase space and the concept of mass. Journal of Physics A: Mathematical and Theoretical, 42, 045204.

Żenczykowski, P. (2014). Elementary particles and emergent phase space. Singapore: World Scientific.

Żenczykowski, P. (2015). Elementary particles, the concept of mass, and emergent spacetime. Journal of Physics: Conference Series, 626(1), 012022.

Żenczykowski, P. (2016). The Harari-Shupe observation without preons-A glimpse of physics to come? Acta Physica Polonica B, 47, 1011-1032. arXiv: 1503.07773.

Żenczykowski, P. (2017). From Clifford algebra of nonrelativistic phase space to quarks and leptons of the Standard Model. Advances in Applied Clifford Algebras, 27, 333-344. 
Piotr Żenczykowski is a professor at the Institute of Nuclear Physics of the Polish Academy of Sciences. He has been associated with Krakow's academic milieu since his MSc studies, followed by a PhD in theoretical physics (1977) also from the Jagiellonian University. After habilitation he spent several years doing research and teaching at the University of Guelph, Ontario, Canada. Throughout his career he has studied the phenomenology of strong and weak interactions of elementary particles, with particular focus on the spectroscopy and weak decays of baryons. With growing realization of the crucial role of physical assumptions, his interests have broadened to include foundational issues and philosophical arguments. These helped to form his ideas on the origin of the Standard Model. Apart from many scientific publications in international peerreviewed journals, he is the author of "Elementary Particles and Emergent Phase-Space" (World Scientific, 2014). 